\title{
Artroplastia Reversa do Ombro: Indicações Emergentes e Resultados
}

\author{
Reverse Shoulder Arthroplasty: \\ Emerging Indications and Results \\ Roberto Barreto Maia ${ }^{1}$, Rogério Meira Barros ${ }^{1}$, Lucas Fraga Cunha da Silva ${ }^{1}$, \\ Marcus Vinícius Silva Santos ${ }^{1}$ \\ ${ }^{1}$ Serviço de Ortopedia e Traumatologia do Hospital Santa Izabel; Salvador, Bahia, Brasil
}

Correspondence addresses:

Dr. Roberto B. Maia

rbmaia@superig.com.br

Received: April 14, 2019

Revised: May 31, 2019

Accepted: June 11, 2019

Published: June 28, 2019

Data Availability Statement: All relevant data are within the paper and its Supporting Information files.

Funding: This work was the result of authors' initiative. There was no support of research or publication funds.

Competing interests: The authors have declared that no competing interests exist.

Copyright

(C) 2019 by Santa Casa de Misericórdia da Bahia.

All rights reserved.

ISSN: 2526-5563
A artroplastia total do ombro e a hemiartroplastia do ombro têm sido o método tradicional para tratar uma variedade de condições do ombro, incluindo artrose glenoumeral, artropatia do manguito rotador e alguns tipos de fraturas. No entanto, esses procedimentos não fornecem resultados consistentes quando empregadas no tratamento de pacientes com ruptura do manguito rotador. $O$ desenvolvimento da artroplastia reversa por Grammont no final do século XX revolucionou o tratamento da osteoartrose glenoumeral associada a rupturas do manguito rotador. A principal indicação para a artroplastia reversa é para o paciente portador de artropatia do manguito rotador apresentando dor e perda do arco de movimento do ombro. Devido aos bons resultados obtidos no tratamento desta patologia, as indicações para utilização da artroplastia reversa se expandiram gradualmente incluindo outras condições que anteriormente eram difíceis de tratar com sucesso e de forma previsível. Esta revisão discute e analisa criticamente essas novas indicações para a artroplastia reversa do ombro.

Palavras-chave: Ombro; Artroplastia; Artrose; Fratura; Cirurgia.

Complete shoulder arthroplasty and shoulder hemiarthroplasty have been the traditional method for treating various shoulder conditions, including glenohumeral arthrosis, rotator cuff arthropathy, and some types of fractures. However, these procedures do not provide consistent results when used in the treatment of patients with rotator cuff rupture. The development of reverse arthroplasty by Grammont in the late $20^{\text {th }}$ century revolutionized the treatment of glenohumeral osteoarthritis associated with rotator cuff ruptures. The main indication for reverse arthroplasty is for the patient with rotator cuff arthropathy presenting with pain and loss of the arch of the shoulder. Due to the good results obtained in the treatment of this condition, indications for the use of reverse arthroplasty gradually expanded, including other conditions that were previously difficult to treat successfully and predictably. This review discusses and analyze these new indications for reverse shoulder arthroplasty.

Keywords: Shoulder; Arthroplasty; Osteoarthritis; Fracture; Surgery; Reverse.

\section{Introdução}

A artroplastia total e a hemiartroplastia do ombro têm sido o método tradicional utilizado para tratar uma variedade de condições do ombro, incluindo artrose glenoumeral, artropatia do manguito e alguns tipos de fraturas. No entanto, esses procedimentos não fornecem resultados 
consistentes, pois em ombros que apresentam rupturas maciças do manguito rotador, as forças que normalmente neutralizam o componente ascendente do deltoide e estabilizam o centro de rotação do ombro estão ausentes.

Existem inúmeras situações nas quais a artroplastia total do ombro (artroplastia convencional) não consegue atingir as expectativas do tratamento. Dentre as patologias, podemos citar: fraturas complexas do úmero proximal, artrose glenoumeral com rupturas do manguito rotador associada, consolidação viciosa do úmero proximal, pseudoparalisia, dentre outras patologias. A artroplastia total não consegue reproduzir a biomecânica do ombro com os pares de força do manguito rotador, levando à perda do arco de movimento, escape antero superior da cabeça umeral e severa limitação funcional. ${ }^{1}$

Em 1987, Paul Grammont apresentou um novo conceito de artroplastia do ombro, a artroplastia reversa. Seu novo design baseava-se em quatro princípios chaves: estabilidade intrínseca da prótese, concavidade da região de suporte e convexidade da região de carga (glenoide), centro da glenoesfera na glenoide ou no interior do colo glenoide e, medialização e distalização do centro de rotação.

A artroplastia reversa do ombro foi originalmente criada para o tratamento da artropatia do manguito rotador. As indicações cirúrgicas para este grupo de paciente incluem dor, perda de movimento, e limitação funcional, quadro clínico este, principalmente, devido à artrose e a não funcionalidade do manguito rotador. Biomecanicamente, a artroplastia reversa proporciona um fulcro estável e fixo, aumentando o braço de alavanca e o tônus muscular em repouso do músculo deltoide. Como resultado, a artroplastia reversa, geralmente, melhora o arco de movimento em abdução e elevação a despeito de um manguito rotador não funcionante. ${ }^{1-4}$

Devido ao tratamento bem-sucedido da artropatia do manguito rotador utilizando a artroplastia reversa, as indicações para o seu uso foram gradualmente aumentando, incluindo, também, tumores do úmero proximal, sequelas de fraturas doúmero proximal, osteartrose com perda óssea importante da glenoide, luxação inveterada do ombro, revisão cirúrgica de hemiartroplastia e de artroplastia total do ombro..$^{5-7}$

Neste artigo de atualização, deu-se ênfase às indicações menos frequentes da artroplastia reversa do ombro, bem como, foi realizada uma revisão da literatura, relatando resultados clínicos e funcionais existentes até o momento.

\section{Rupturas Irreparáveis do Manguito Rotador sem Osteoartrose Glenoumeral}

Existe um grupo de pacientes com rupturas maciças do manguito rotador em que o principal problema é a perda do arco de movimento. Os pacientes em questão não conseguem elevar o braço acima do nível do ombro devido à fraqueza gerada pela ruptura do manguito ou ao escape antero superior da cabeça umeral que não consegue permanecer centralizada na glenoide durante a elevação do braço. Esta condição é chamada de pseudoparalisia devido ao severo impacto funcional.

A artroplastia reversa é um tratamento bastante utilizado em pacientes com pseudoparalisia do ombro, secundária á ruptura maciça irreparável do manguito rotador. Vários estudos sustentam esse tipo de abordagem cirúrgica nesta patologia., ${ }^{5,8}$, Wall e colaboradores ${ }^{4}$ não encontraram diferenças entre pacientes portadores de rupturas maciças do manguito com osteartrose glenoumeral e sem osteoartrose.

Portanto, a artroplastia reversa do ombro é uma opção de tratamento para este grupo de pacientes com resultados consistentes, obtendo a melhora do arco de movimento comparado às medidas pré-operatórias.

\section{Fraturas Complexas do Úmero Proximal}

Recentemente, estudos com follow-up longo têm demonstrado limitações e altas taxas de complicações utilizando a hemiartroplastia para tratamento de fraturas complexas do úmero 
proximal, dentre elas, diminuição progressiva do espaço acromioumeral, osteólise ao redor da haste umeral e reabsorção das tuberosidades. ${ }^{10,11}$

Autilizaçãodeartroplastiareversaempacientes com fraturas complexas do úmero proximal, ao contrário da hemiartroplastia, não é totalmente dependente da consolidação das tuberosidades, nem da integridade do manguito rotador. Pacientes tratados com artroplastia reversa apresentam recuperação mais rápida comparado àqueles submetidos à hemiartroplastia. Estudos recentes demostram resultados mais previsíveis em pacientes submetidos à artroplastia reversa do ombro. ${ }^{12-19}$

Boileau e colaboradores ${ }^{20}$, avaliando 39 ombros em pacientes, com média de idade de 77 anos e follow-up de 83 meses, alertam para o reparo das tuberosidades do úmero proximal e, a despeito da idade avançada e osteopenia, a consolidação obtida minimiza as incidências de complicações pós-operatórias, otimiza a função do manguito rotador e promove melhor rotação externa ativa. Gigis e colaboradores ${ }^{21}$ mostraram que o uso da artroplastia reversa do ombro para tratamento de fraturas do úmero proximal, especialmente em pacientes com idade superior a 70 anos, traz inúmeros benefícios, dentre eles: menor tempo de reabilitação e preservação de fulcro fixo e estável para manutenção da função do deltoide em caso de falha do manguito rotador. Quando compararam os resultados da artroplastia reversa com hemiartroplastia, os autores encontraram resultados favoráveis à primeira, porém alertam para a necessidade de experiência do cirurgião com o uso da técnica e importância de indicações precisas com a finalidade de minimizar complicações.

Embora alguns estudos tenham demonstrado bons resultados utilizando a artroplastia reversa para o tratamento de fraturas do úmero proximal, outros têm relatado resultados inferiores àqueles esperados tanto pelo cirurgião quanto pelo paciente. Smith e colaboradores ${ }^{7}$ relataram resultados inferiores em pacientes tratados devido a fraturas do úmero proximal quando comparados àqueles portadores de artropatia do manguito rotador. Essa conclusão foi baseada em medidas de elevação anterior de aproximadamente $100^{\circ} \mathrm{e}$ uma grande variação na rotação interna e externa do ombro (Figura 1).

Figura 1. Fratura grave do úmero proximal e artroplastia reversa utilizada para tratamento.

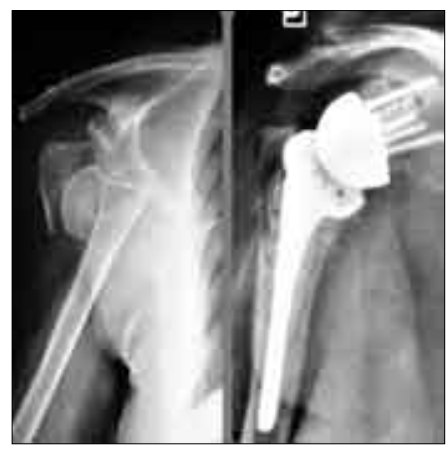

\section{Consolidação Viciosa em Fraturas do Úmero Proximal}

Fraturas do úmero proximal consolidadas viciosamente apresentam assimetria da anatomia óssea, bem como, degeneração gordurosa da musculatura do manguito rotador. A artroplastia total utilizada para o tratamento específico desta enfermidade apresenta taxas de falha muito elevadas. $^{22-24}$ Devido à menor dependência das tuberosidades e do manguito rotador, a artroplastia reversa tem sido usada para tratamento das fraturas viciosamente consolidadas e pseudoartroses do úmero proximal, apresentando resultados satisfatórios. $^{25,26}$

Willis e colaboradores ${ }^{22}$ relataram que a artroplastia reversa do ombro pode ser utilizada para tratamento das mais variadas consolidações viciosas e pseudoartroses do úmero proximal, ao contrário das próteses não constrictas. Kilic e colaboradores ${ }^{25}$ compararam o uso da artroplastia total e artroplastia reversa para o tratamento de fraturas viciosamente consolidadas do úmero proximal e demonstraram resultados clínicos favoráveis ao uso da artroplastia reversa nesta condição.

Embora a artroplastia reversa apresente superioridade em relação à artroplastia total convencional para o tratamento de consolidação 
viciosa, os resultados clínicos obtidos são inferiores aos relatados quando utilizada para tratamento de artropatia do manguito rotador (Figuras 2A-2C).

\section{Osteoartrose Glenoumeral com Perda Óssea da Glenoide}

Perda óssea grave da glenoide pode ser observada em inúmeras situações, tais como, falhas em artroplastia do ombro, osteoartrose de origem inflamatória, osteoartrose primária com instabilidade posterior e luxações inveteradas da articulação glenoumeral. ${ }^{27-30}$

Boileau e colaboradores ${ }^{31}$ avaliaram 54 pacientes submetidos à artroplastia reversa do ombro, utilizando enxerto ósseo autólogo da cabeça umeral (BIO-reversa) com follow-up mínimo de 2 anos em ombros que apresentavam defeitos variados da glenoide, retroversão acentuada e deformidades em mais de um plano. Os autores mostraram que a utilização da BIO-reversa consegue corrigir as deficiências glenoidais, mesmo em casos severos $\left(>25^{\circ}\right)$ e com deformidades multiplanares, além de apresentar incorporação do enxerto ósseo em 94\% dos casos.

A perda óssea da glenoide é um problema frequentemente encontrado durante a realização da artroplastia reversa e difícil de ser abordado. A não identificação e falta de planejamento para a abordagem da perda óssea pode levar ao posicionamento inadequado do componente glenoidal e consequentemente complicações e falhas do procedimento. Seidl e colaboradores ${ }^{32}$ demonstram, em artigo de revisão, inúmeras técnicas para abordagem da perda óssea da glenoide utilizando a artroplastia reversa, dentre elas: fresagem assimétrica da glenoide e uso de implantes lateralizados, enxertia óssea e uso de implantes (baseplate) com compensações.

A vantagem da prótese reversa é que esta pode ser implantada, na maioria dos casos, em pacientes que apresentam importante perda óssea da glenoide, principalmente, utilizando os implantes que possuem fixação através de um parafuso central, o que não é possível com o uso da artroplastia total.

\section{Revisão de Artroplastia Total e Hemi- artroplastia do Ombro}

A artroplastia total do ombro tem sido utilizada com grande frequência como opção para cirurgias de revisão, porém muitos estudos têm demonstrado resultados insatisfatórios, ${ }^{33-35}$ especialmente devido á disfunção de partes moles ou soltura do componente glenoidal, instabilidade, ou infecção. ${ }^{36,37}$

Diante desses resultados, o uso da prótese reversa do ombro tem aumentado como procedimento de revisão após falhas das artroplastias (Figuras 3 e 4).

Figura 2. A. Consolidação viciosa do úmero proximal com artrose glenoumeral. B. Artroplastia reversa do ombro para tratamento de consolidação viciosa. C. Artroplastia reversa do ombro para tratamento de consolidação viciosa. D. Resultado clínico após tratamento de consolidação viciosa com artroplastia reversa. Nota-se elevação anterior ativa de $140^{\circ}$ e rotação interna ao nível do glúteo.
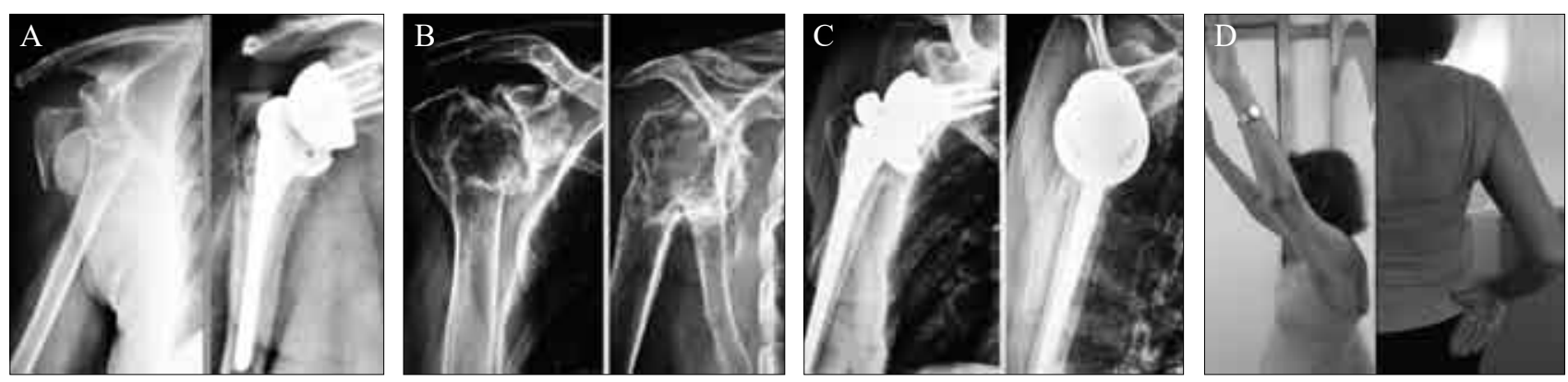
Figura 3. Falha de artroplastia total do ombro e implante retirado durante revisão cirúrgica.

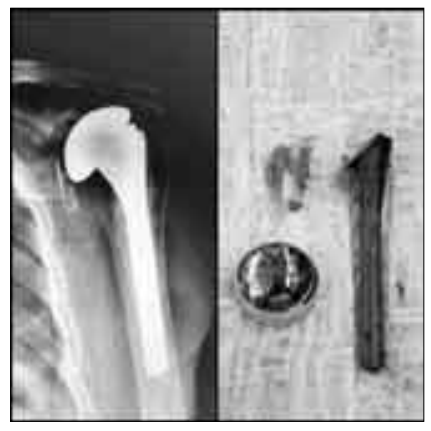

Figura 4. Tratamento de falha de artroplastia total utilizando artroplastia reversa do ombro.

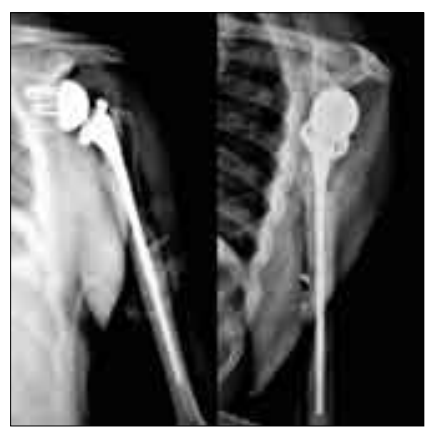

Flury e colaboradores ${ }^{38}$ obtiveram resultados estatisticamente significantes quando comparou o arco de movimento pré-operatório e pósoperatório em pacientes submetidos à artroplastia reversa de revisão, porém a elevação anterior ativa encontrada foi de aproximadamente $90^{\circ}$, valores muito inferiores quando comparados à artroplastia reversa primária.

Jo Sh e colaboradores ${ }^{39}$ concluíram que procedimentos de revisão utilizando a artroplastia reversa proporcionam alívio da dor. Entretanto, a melhora da mobilidade e função são difíceis de serem recuperadas. E, embora a função e arco de movimento melhorem com relação aos valores préoperatórios, estes não se mostraram tão significantes quanto esperados. Apesar destes achados, os pacientes ficaram satisfeitos com os resultados obtidos, possivelmente devido à severidade da dor e limitações do arco de movimento no préoperatório.

Embora a artroplastia reversa seja uma boa opção para cirurgias de revisão após falha de outras artroplastias, existe grande preocupação com relação ao número elevado de complicações e resultados inferiores quando comparados à utilização dessa prótese em procedimentos primários. ${ }^{7}$

\section{Displasias do Ombro}

A displasia da glenoide pode ocorrer como uma condição isolada ou em associação com outras patologias, tais como, displasias epifisárias, síndrome de Kniest, artrogripose, trauma obstétrico, infecção e distrofia muscular. ${ }^{40}$ Há pouca informação disponível a respeito da artroplastia reversa no tratamento de pacientes com displasia da glenoide devido à raridade dessa condição.

\section{Luxações Inveteradas do Ombro (Anterior e Posterior)}

Em pacientes com luxação crônica do ombro, além de rupturas do manguito rotador e grave comprometimento da arquitetura óssea da cabeça umeral glenoide, pode-se encontrar osteoporose, amolecimento da cartilagem articular e severa contratura de partes moles. ${ }^{41,42}$ A utilização da artroplastia total no tratamento destes pacientes não mostrou resultados satisfatórios. ${ }^{43}$

Aliteratura é escassa com relação ao tratamento desta patologia utilizando a artroplastia reversa do ombro. Hyun e colaboradores ${ }^{44}$ relataram resultados promissores com o uso da prótese reversa do ombro em pacientes com luxações inveteradas. O grupo de ombro da Santa Casa de Misericórdia da Bahia tratou cinco pacientes portadores desta patologia e, embora os resultados não tenham sido publicados, todos apresentaram melhora do quadro álgico e arco de movimento em comparação aos valores pré-operatórios e os resultados foram muito satisfatórios (Figuras 5 e 6 ).

\section{Artrites Autoimunes com ou sem Rupturas do Manguito Rotador}

Várias doenças autoimunes podem ter manifestações articulares e, muitas delas acometem a articulação glenoumeral, tais como, 
Figura 7. Luxação inveterada do ombro.

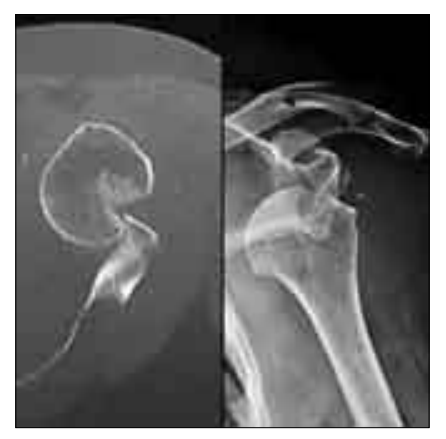

Figura 8. Artroplastia reversa implantada para tratamento de luxação inveterada do ombro.

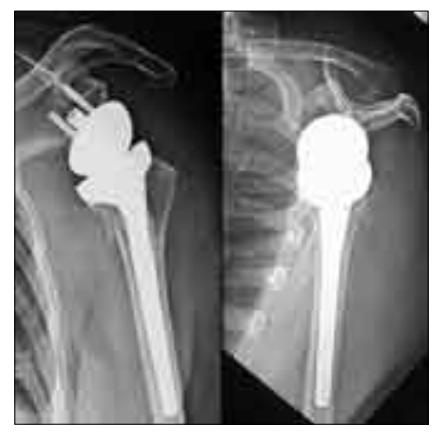

artrite reumatoide, doença inflamatória de Bowel, esclerodermia e espondilite anquilosante. Embora a artroplastia total seja utilizada em pacientes com artrite reumatoide e apresente bons resultados em relação ao alívio da dor, a melhora do arco de movimento e função são menos satisfatórios. ${ }^{45-48} \mathrm{~A}$ destruição articular causada pela artrite reumatoide é, geralmente, associada à deficiência do manguito rotador devido à sua disfunção ou franca ruptura. ${ }^{49} \mathrm{~A}$ falha deste resulta em migração superior da cabeça umeral, sendo este problema frequentemente observado após o tratamento da afecção utilizando hemiartroplastia ou artroplastia total do ombro..$^{50,51}$

Young e colaboradores ${ }^{49}$ relataram que a artroplastia reversa do ombro deveria ser o procedimento de escolha para tratamento de pacientes portadores de artrite reumatoide com mais de 65 anos, independente da integridade do manguito rotador.

Em revisão sistemática publicada recentemente, Cho e colaboradores ${ }^{52}$ mostraram que a utilização da prótese reversa em pacientes portadores de artrite reumatoide apresenta resultados e complicações semelhantes quando comparados àqueles com diagnóstico de artropatia do manguito rotador submetidos ao mesmo tratamento.

O uso da artroplastia reversa em pacientes com artrite reumatoide é objeto de avaliação em diversos estudos, ${ }^{53,54}$ demonstrando melhora significativa da dor, bem como, arco de movimento e função em pacientes submetidos a este procedimento cirúrgico. ${ }^{54,55}$

\section{Considerações Finais}

A artroplastia reversa do ombro tem se mostrado um procedimento muito eficaz para tratamento de pacientes com artrose do ombro associada à ruptura do manguito rotador. Devido aos bons resultados encontrados nesse tipo de população, suas indicações têm sido gradualmente ampliadas para uma grande variedade de patologias e condições particulares do ombro. Para a maioria destas novas indicações cirúrgicas, a artroplastia reversa tem obtido excelente resultados, porém é necessário um maior seguimento para avaliação de complicações e resultados a longo prazo.

\section{Referências}

1. Edwards TB, Boulahia A, Kempf JF, Boileau P, Nemoz $\mathrm{C}$, Walch $\mathrm{G}$. The influence of rotator cuff disease on the results of shoulder arthroplasty for primary osteoarthritis: results of a multicenter study. J Bone Joint Surg Am. 2002;84(12):2240-8.

2. Nolan BM,Ankerson E, Wiater JM. Reverse total shoulder arthroplasty improves function in cuff tear arthropathy. Clin Orthop Relat Res. 2011;469(9):2476-82.

3. Gerber C, Pennington SD, Nyffeler RW. Reverse total shoulder arthroplasty. J Am Acad Orthop Surg. 2009;17(5):284- 95.

4. Khan WS, Longo UG, Ahrens PM, Denaro V, Maffulli $\mathrm{N}$. A systematic review of the reverse shoulder replacement in rotator cuff arthropathy, rotator cuff tears, and rheumatoid arthritis. Sports Med Arthrosc. 2011;19(4):366-79.

5. Wall B, Nove-Josserand L, O'Connor DP, Edwards TB, Walch G. Reverse total shoulder arthroplasty: a review of results according to etiology. J Bone Joint Surg Am. 2007;89(7):1476-85. 
6. Austin L, Zmistowski B, Chang ES, Williams GR Jr. Is reverse shoulder arthroplasty a reasonable alternative for revision arthroplasty? Clin Orthop Relat Res. 2011;469(9):2531- 7.

7. Smith CD, Guyver P, Bunker TD. Indications for reverse shoulder replacement: a systematic review. J Bone Joint Surg Br. 2012;94(5):577-83.

8. Boileau P, Gonzalez JF, Chuinard C, Bicknell R, Walch G. Reverse total shoulder arthroplasty after failed rotator cuff surgery. J Shoulder Elbow Surg. 2009;18(4):600-6.

9. Mulieri P, Dunning P, Klein S, Pupello D, Frankle M. Reverse shoulder arthroplasty for the treatment of irreparable rotator cuff tear without glenohumeral arthritis. J Bone Joint Surg Am. 2010;92(15):2544-56.

10. Antuna SA, Sperling JW, Cofield RH. Shoulder hemiarthroplasty for acute fractures of the proximal humerus: a minimum five-year follow-up. J Shoulder Elbow Surg. 2008;17(2):202-9.

11. Noyes MP, Kleinhenz B, Markert RJ, Crosby LA. Functional and radiographic long-term outcomes of hemiarthroplasty for proximal humeral fractures. J Shoulder Elbow Surg. 2011;20(3):372-7.

12. Lenarz C, Shishani Y, McCrum C, Nowinski RJ, Edwards TB, Gobezie R. Is reverse shoulder arthroplasty appropriate for the treatment of fractures in the older patient? Early observations. Clin Orthop Relat Res. 2011;469(12):3324-31.

13. BoyleMJ, Youn SM, Frampton CM, BallCM. Functional outcomes of reverse shoulder arthroplasty compared with hemiarthroplasty for acute proximal humeral fractures. J Shoulder Elbow Surg. 2013;22(1):32-7.

14. Bufquin T, Hersan A, Hubert L, Massin P. Reverse shoulder arthroplasty for the treatment of three- and fourpart fractures of the proximal humerus in the elderly: a prospective review of 43 cases with a short-term followup. J Bone Joint Surg Br. 2007;89(4):516-20.

15. Cazeneuve JF, Cristofari DJ. The reverse shoulder prosthesis in the treatment of fractures of the proximal humerus in the elderly. J Bone Joint Surg Br. 2010;92(4):535-9.

16. Gallinet D, Clappaz P, Garbuio P, Tropet Y, Obert L. Three or four parts complex proximal humerus fractures: hemiarthroplasty versus reverse prosthesis: a comparative study of 40 cases. Orthop Traumatol Surg Res. 2009;95(1):48-55.

17. Garrigues GE, Johnston PS, Pepe MD, Tucker BS, Ramsey ML, Austin LS. Hemiarthroplasty versus reverse total shoulder arthroplasty for acute proximal humerus fractures in elderly patients. Orthopedics 2012;35(5):e703-8.

18. Klein M, Juschka M, Hinkenjann B, Scherger B, Ostermann PA. Treatment of comminuted fractures of the proximal humerus in elderly patients with the Delta III reverse shoulder prosthesis. J Orthop Trauma. 2008;22(10):698-704.
19. Young SW, Segal BS, Turner PC, Poon PC. Comparison of functional outcomes of reverse shoulder arthroplasty versus hemiarthroplasty in the primary treatment of acute proximal humerus fracture. ANZ J Surg. 2010;80(11):789-93.

20. Boileau P, Alta TD, Decroocq L, Sirveaux F, Clavert P, Favard L, Chelli M. Reverse shoulder arthroplasty for acute fractures in the elderly: is it worth reattaching the tuberosities? J Shoulder Elbow Surg. 2019 Mar;28(3):437-444. doi: 10.1016/j.jse.2018.08.025. Epub 2018 Dec 18.

21. Gigis I, Nenopoulos A, Giannekas D, Heikenfeld R, Beslikas T, Hatzokos I. Reverse Shoulder Arthroplasty for the Treatment of 3 and 4- Part Fractures of the Humeral Head in the Elderly.Open Orthop J. 2017 Feb 28;11:108-118. doi: 10.2174/1874325001711010108. eCollection 2017. Review

22. Willis M, Min W, Brooks JP, et al. Proximal humeral malunion treated with reverse shoulder arthroplasty. J Shoulder Elbow Surg. 2012;21(4):507-13.

23. Barrett WP, Franklin JL, Jackins SE, Wyss CR, Matsen FA 3rd. Total shoulder arthroplasty. J Bone Joint Surg Am. 1987;69(6):865-72.

24. Franklin JL, Barrett WP, Jackins SE, Matsen FA 3rd. Glenoid loosening in total shoulder arthroplasty: association with rotator cuff deficiency. J Arthroplasty. 1988;3(1):39-46.

25. Kilic M, Berth A, Blatter G, et al. Anatomic and reverse shoulder prostheses in fracture sequelae of the humeral head. Acta Orthop Traumatol Turc. 2010;44(6):417-25.

26. Martinez AA, Calvo A, Bejarano C, Carbonel I, Herrera A. The use of the Lima reverse shoulder arthroplasty for the treatment of fracture sequelae of the proximal humerus. J Orthop Sci. 2012;17(2):141.

27. Beran MC, Donaldson CT, Bishop JY. Treatment of chronic glenoid defects in the setting of recurrent anterior shoulder instability: a systematic review. J Shoulder Elbow Surg. 2010;19(5):769-80.

28. Holcomb JO, Hebert DJ, Mighell MA, et al. Reverse shoulder arthroplasty in patients with rheumatoid arthritis. J Shoulder Elbow Surg. 2010;19(7):1076-84.

29. Neyton L, Boileau P, Nove-Josserand L, Edwards TB, Walch G. Glenoid bone grafting with a reverse design prosthesis. J Shoulder Elbow Surg. 2007;16(3 Suppl):S71-8.

30. Strauss EJ, Roche C, Flurin PH, Wright T, Zuckerman JD. The glenoid in shoulder arthroplasty. J Shoulder Elbow Surg. 2009;18(5):819-33.

31. Boileau P, Morin-Salvo N, Gauci MO, Seeto BL, Chalmers PN, Holzer N, Walch G. Angled BIO-RSA (bony-increased offset-reverse shoulder arthroplasty): a solution for the management of glenoid bone loss and erosion.J Shoulder Elbow Surg. 2017 Dec;26(12):21332142. doi: 10.1016/j.jse.2017.05.024. Epub 2017 Jul 20. 
32. Seidl, A. J., Williams, G. R., \& Boileau, P. (2016). Challenges in Reverse Shoulder Arthroplasty: Addressing Glenoid Bone Loss. Orthopedics, 39(1), 14-23. doi:10.3928/01477447-20160111-01

33. Sassoon AA, Rhee PC, Schleck CD, Harmsen WS, Sperling JW, Cofield RH. Revision total shoulder arthroplasty for painful glenoid arthrosis after humeral head replacement: the nontraumatic shoulder. J Shoulder Elbow Surg. 2012;21(11):1484-91.

34. Hattrup SJ. Revision total shoulder arthroplasty for painful humeral head replacement with glenoid arthrosis. J Shoulder Elbow Surg. 2009;18(2):220-4.

35. Sperling JW, Cofield RH. Revision total shoulder arthroplasty for the treatment of glenoid arthrosis. J Bone Joint Surg Am. 1998;80(6):860-7.

36. Carroll RM, Izquierdo R, Vazquez M, Blaine TA, Levine WN, Bigliani LU. Conversion of painful hemiarthroplasty to total shoulder arthroplasty: long-term results. J Shoulder Elbow Surg. 2004;13(6):599-603.

37. Dines JS, Fealy S, Strauss EJ, et al. Outcomes analysis of revision total shoulder replacement. J Bone Joint Surg Am. 2006;88(7):1494-500.

38. Flury MP, Frey P, Goldhahn J, Schwyzer HK, Simmen BR. Reverse shoulder arthroplasty as a salvage procedure for failed conventional shoulder replacement due to cuff failure--midterm results. Int Orthop. 2011 Jan;35(1):53-60. doi: 10.1007/s00264-010-0990-z. Epub 2010 Mar 14.

39. Jo SH, Kim JY, Cho NS, Rhee YG. Reverse Total Shoulder Arthroplasty: Salvage Procedure for Failed Prior Arthroplasty.Clin Orthop Surg. 2017 Jun;9(2):200-206. doi: 10.4055/cios.2017.9.2.200. Epub 2017 May 8.

40. Wirth MA, Lyons FR, Rockwood CA Jr. Hypoplasia of the glenoid: a review of sixteen patients. J Bone Joint Surg Am. 1993;75(8):1175-84

41. Sahajpal DT, Zuckerman JD. Chronic glenohumeral dislocation. J Am Acad Orthop Surg. 2008;16(7):38598.

42. Sperling JW, Cofield RH, Schleck CD, Harmsen WS. Total shoulder arthroplasty versus hemiarthroplasty for rheumatoid arthritis of the shoulder: results of 303 consecutive cases. J Shoulder Elbow Surg. 2007;16(6):683-90.

43. Checchia SL, Santos PD, Miyazaki AN. Surgical treatment of acute and chronic posterior fracturedislocation of the shoulder. J Shoulder Elbow Surg. 1998;7(1):53-65.
44. Yoon Suk Hyun, MD, Gazi Huri, MD, Nickolas G. Garbis, MD, Edward G. McFarland, MD. Uncommon indications for reverse total shoulder arthroplasty. Clinics in Orthopedic Surgery 2013;(5)4.

45. Betts HM, Abu-Rajab R, Nunn T, Brooksbank AJ. Total shoulder replacement in rheumatoid disease: a 16- to 23- year follow-up. J Bone Joint Surg Br. 2009;91(9):1197-200.

46. Boyd AD Jr, Thomas WH, Scott RD, Sledge CB, Thornhill TS. Total shoulder arthroplasty versus hemiarthroplasty: indications for glenoid resurfacing. J Arthroplasty. 1990;5(4):329-36.

47. Sneppen O, Fruensgaard S, Johannsen HV, Olsen BS, Sojbjerg JO, Andersen NH. Total shoulder replacement in rheumatoid arthritis: proximal migration and loosening. J Shoulder Elbow Surg. 1996;5(1):47-52.

48. Trail IA, Nuttall D. The results of shoulder arthroplasty in patients with rheumatoid arthritis. J Bone Joint Surg Br. 2002;84(8):1121-5.

49. Young AA, Smith MM, Bacle G, Moraga C, Walch G. Early results of reverse shoulder arthroplasty in patients with rheumatoid arthritis. J Bone Joint Surg Am. 2011;93(20):1915-23.

50. Khan A, Bunker TD, Kitson JB. Clinical and radiological follow-up of the Aequalis third-generation cemented total shoulder replacement: a minimum tenyear study. J Bone Joint Surg Br. 2009;91(12):1594600.

51. Stewart MP, Kelly IG. Total shoulder replacement in rheumatoid disease: 7- to 13-year follow-up of 37 joints. J Bone Joint Surg Br. 1997;79(1):6872.

52. Cho CH, Kim DH, Song KS. Reverse Shoulder Arthroplasty in Patients with Rheumatoid Arthritis: A Systematic Review. Clin Orthop Surg. 2017 Sep;9(3):325-331. doi: 10.4055/cios.2017.9.3.325. Epub 2017 Aug 4. Review.

53. Ekelund A, Nyberg R. Can reverse shoulder arthroplasty be used with few complications in rheumatoid arthritis? Clin Orthop Relat Res. 2011;469(9):2483-8.

54. Rittmeister M, Kerschbaumer F. Grammont reverse total shoulder arthroplasty in patients with rheumatoid arthritis and non reconstructible rotator cuff lesions. J Shoulder Elbow Surg. 2001;10(1):17-22.

55. Woodruff MJ, Cohen AP, Bradley JG. Arthroplasty of the shoulder in rheumatoid arthritis with rotator cuff dysfunction. Int Orthop. 2003;27(1):7-10. 